\title{
Group Visits: A Qualitative Review of Current Research
}

\author{
Raja Jaber, MD, Amy Braksmajer, $M P H$, and Jeffrey S. Trilling, $M D$
}

Purpose: The group visit model has emerged as one possible solution to problems posed by the limitations of current structures of care and the demands of a growing chronic illness load. In this article, we summarize current group visit research and develop suggestions for furthering this care model.

Methods: An electronic review of all group visit articles published from the years 1974 to 2004 was conducted via the PubMed and MedLine databases. Reference sections of articles thus obtained were mined for additional citations. Articles were excluded if: (1) they were not research studies (ie, purely descriptive, with no evaluative component); or (2) the group visit intervention was subsumed under larger primary or hospital-based interventions.

Results: Although the heterogeneity of the studies presented renders the assessment of this care model problematic, there is sufficient data to support the effectiveness of group visits in improving patient and physician satisfaction, quality of care, quality of life, and in decreasing emergency department and specialist visits.

Conclusion: Group visits are a promising approach to chronic care management for the motivated patient. Future research may benefit, however, from abandoning old nomenclatures and clearly defining the structure, processes of care, content of visits, and appropriate outcome measures. (J Am Board Fam Med 2006;19:276-90.)

Over the past 2 decades, chronic illness has become a pressing public health issue. Over 90 million Americans suffer from a chronic disease, accounting for more than $75 \%$ of the nation's total medical care costs. ${ }^{1}$ In the United States, $70 \%$ of deaths are secondary to chronic illness ${ }^{1}$; in addition, chronic illness is a leading cause of morbidity, disability, pain, and reduction in quality of life..$^{2-5}$ Medicine's historic focus on the treatment of acute illness has rendered many physicians ill-equipped to handle this influx of chronically ill patients, who usually require complex services and in-depth case management. $^{6}$

The group visit model has emerged as one possible solution to problems posed by the limitations of current care structures relating to chronic illness

Submitted 11 March 2005; revised 14 November 2005; accepted 17 November 2005.

From the State University of New York at Stony Brook, Department of Family Medicine, School of Medicine, Stony Brook, NY.

Conflict of interest: none declared.

Corresponding author: Raja Jaber, MD, Department of Family Medicine, School of Medicine, Health Science Center, Level 4, SUNY at Stony Brook, Stony Brook, NY 11794-8461 (E-mail: rjaber@notes.cc.sunysb.edu). management. Originally conceived in 1974 as a model for well-child consultations, ${ }^{7}$ group visits are mentioned as one of the 10 features of the Future of Family Medicine Project's New Model of Family Medicine ${ }^{8}$ and have recently been popularized in practice management literature. $^{9-14}$

Group visits include most components of individual visits, usually including private or semiprivate one-on-one medical evaluations conducted by a physician or nurse practitioner at each visit, as well as group educational sessions that emphasize patient self-management and address topics such as medical and pharmaceutical management, nutrition, exercise, and psychosocial contributors to health and illness. The time spent with each patient is therefore increased while maintaining or sometimes increasing profitability. ${ }^{11,15,16}$ Group visits, with the exception of drop-in group medical appointments (DIGMAs), are usually diagnosis- or population-specific (ie, diabetics or frail elderly at high risk for hospitalization), comprise the same cohort of patients from visit to visit, and usually include separate private individual medical consultations. ${ }^{9,15}$ In contrast, DIGMAs are meant to address problems of patient access and are composed 
of different patients from meeting to meeting who participate when they have a specific medical need. ${ }^{9,17}$ DIGMAs always include a mental health specialist or social worker that addresses the emotional sequelae of chronic illness, and include an individual medical visit conducted within the group, rather than privately.

Education sessions may be didactic, interactive, or a combination of the 2 , and include various levels of skill building and collaboration with other professionals. Group visits vary in length (sessions may range from 1 hour to half a day) and frequency, meeting once (headache clinics), weekly (high risk cohorts), monthly (cooperative health care clinics (CHCCs), high risk cohorts, and cluster visits), or quarterly (chronic care clinics that usually last half a day). Programs may last for one session or continue over a number of years.

Group visits seem particularly suited to chronic illness management in that they allow more time for self-management education, skill-building, and doctor-patient interaction. Group education reinforces messages received in the individual medical visit, increases perceived benefits, and provides social persuasion and effective action cues. ${ }^{18}$ Group problem solving and social support may also reduce perceived barriers to behavior change. Group visits may reinforce patients' self-efficacy (ie, judgment of their capabilities to carry out the specific tasks necessary to achieve a desired goal ${ }^{19}$ ), which is itself strongly associated with successful chronic disease self-management ${ }^{20}$ Modeling, or seeing that others have accomplished the desired behavior and overcome obstacles, is another powerful contributor to patient self-efficacy. ${ }^{18}$

We have been providing group visits for chronically ill patients since 2001. Our fee-for-service practice currently offers group visits for asthma, lipid management, menopause, and osteoporosis that are based on the high-risk cohort model, meet weekly over the course of 3 or 4 weeks, and are led by a physician/nurse practitioner team. ${ }^{21}$

In this article, we (1) systematically summarize current group visit research to familiarize the reader with this adjunctive model of chronic care management; (2) critically assess the impact of this model on various health outcomes; and (3) offer suggestions for clarifying the structures, processes, and content of this line of care in the hopes of facilitating future research.

\section{Methods}

The authors conducted a systematic, electronic review of the literature from the years 1974 to 2004 via the PubMed and MedLine databases. Search terms included "group visit(s)" and "cluster visit(s)"; the search had to be restricted to article titles because of the extreme commonality of the terms "group" and "visit(s)." Furthermore, articles were obtained by reviewing bibliographies of articles gathered through our database search. This search produced 33 articles. Articles were reviewed in full for descriptions of the target population and intervention, research design and methods, outcomes studied, rates of attendance, and study quality. Articles were excluded if: (1) they were not research studies (ie, purely descriptive, with no evaluative component) $6,7,9,11-13,17,22-26$; or (2) the group visit intervention was subsumed under larger primary or hospital-based interventions. ${ }^{27}$ Finally, 2 articles were excluded because they were subanalyses of data that were covered in greater detail in other studies. ${ }^{28,29}$ After excluding these articles, 18 articles remained for review, 3 of which were reports of the same study. ${ }^{30-32} \mathrm{We}$ included prospective observational studies and randomized controlled clinical trials. The control group always referred to usual care except for Masley's, where it referred to usual care and nutritional educational handouts. ${ }^{33}$

Several research studies that claimed to examine the effects of "group visits" did not include one-onone medical assessments in addition to group education sessions in their protocol ${ }^{33}$ or, alternatively, provided the individual visit as needed, ${ }^{34-38}$ although the addition of this individualized component is what differentiates group visits from educational workshops and peer-led support and self-management groups.

We organized this qualitative review by sequentially describing the effect of all reviewed interventions on each of the following health outcomes (if measured): patient satisfaction, health services utilization, quality of care, health behaviors, physical function/depression/quality of life, disease-specific outcomes, physician satisfaction, and cost of care. Over the course of the review, we were confronted with organizing a large body of heterogeneous information, including various chronic conditions, populations, program lengths, follow-up frequencies, study durations, group visit models used, research designs, and patient attendance rates. We have summarized these differences in Table 1, both 


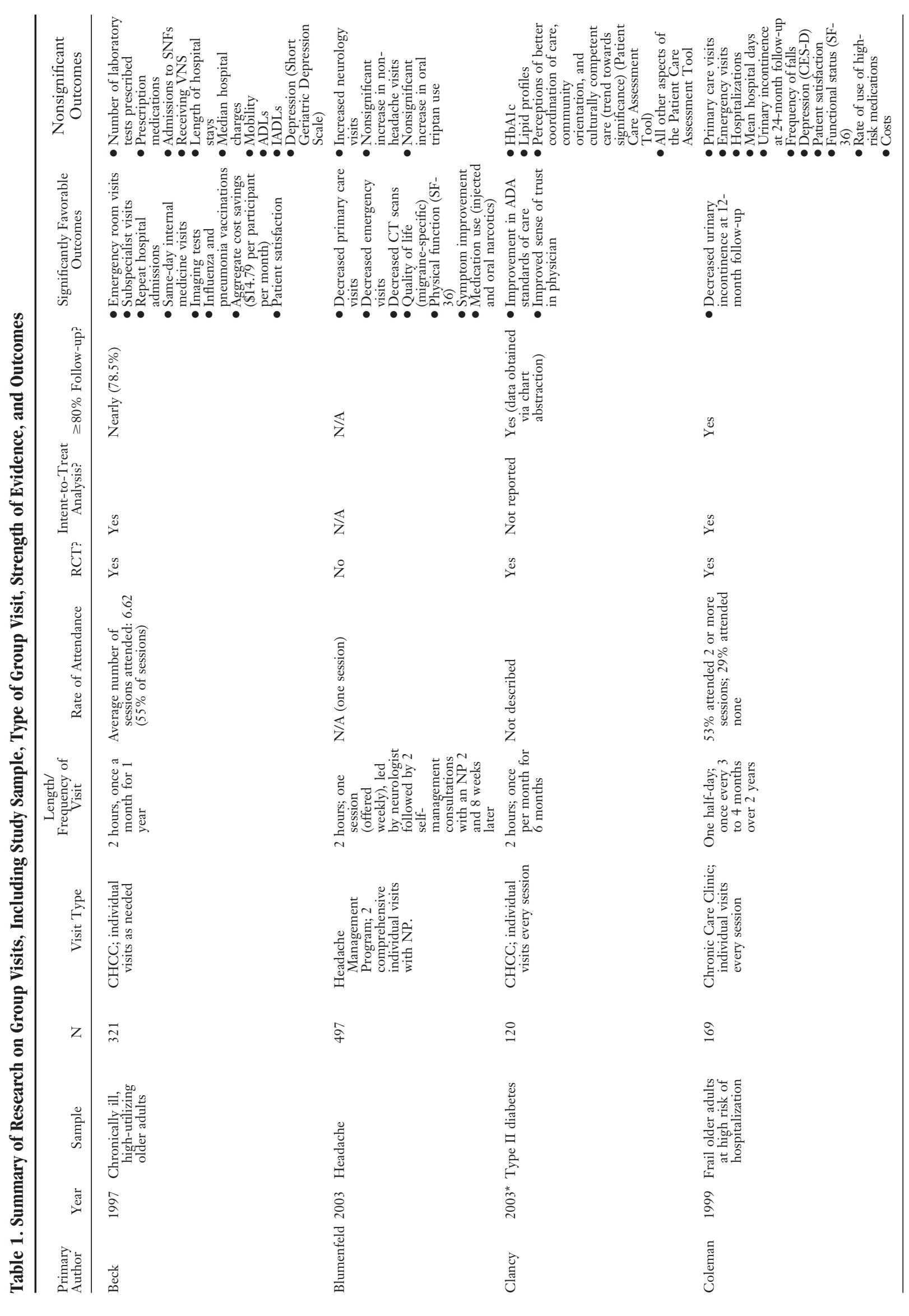











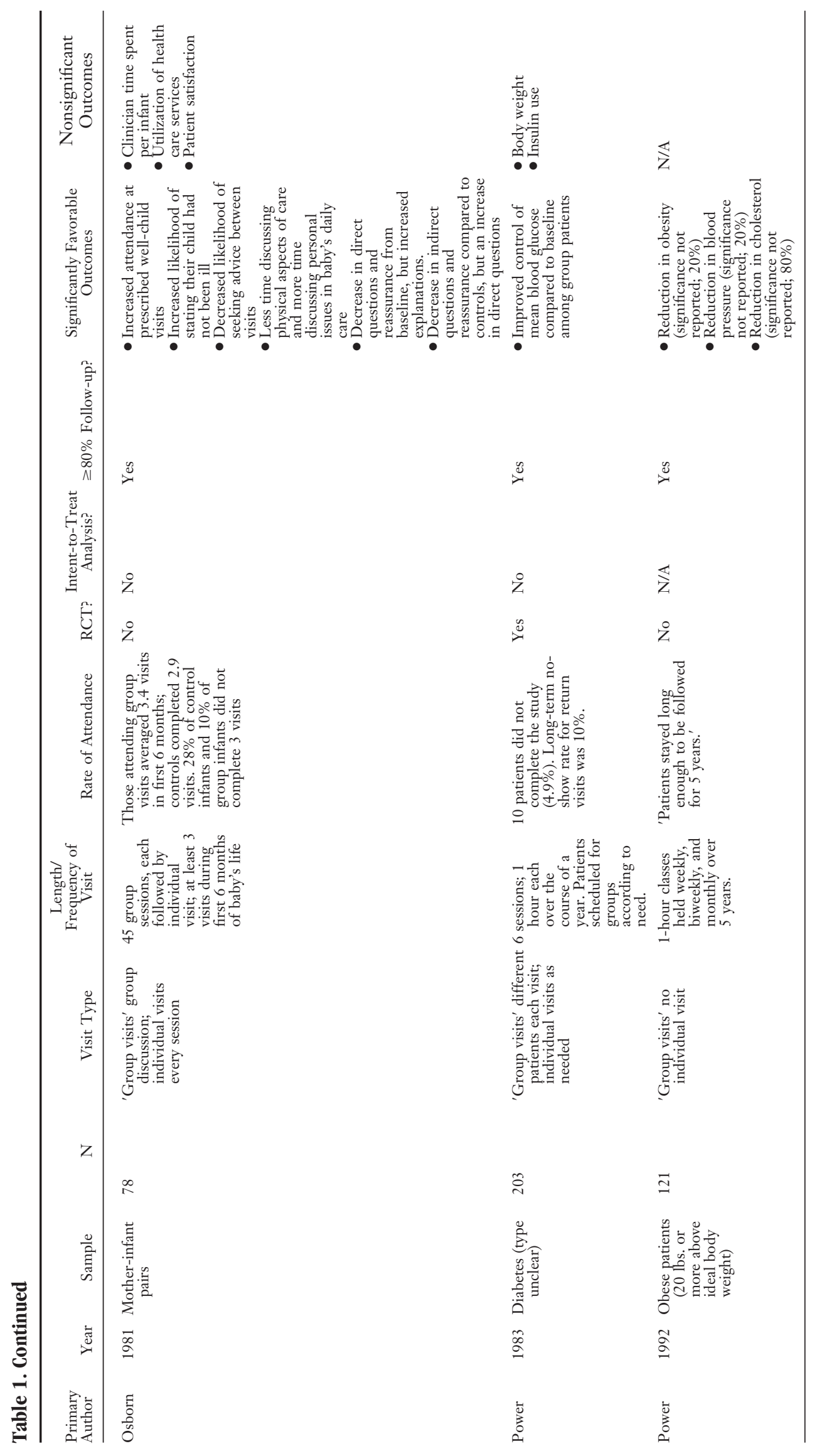

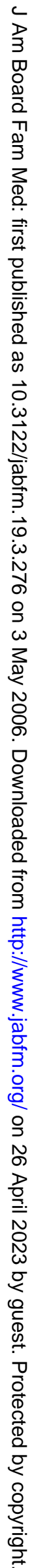









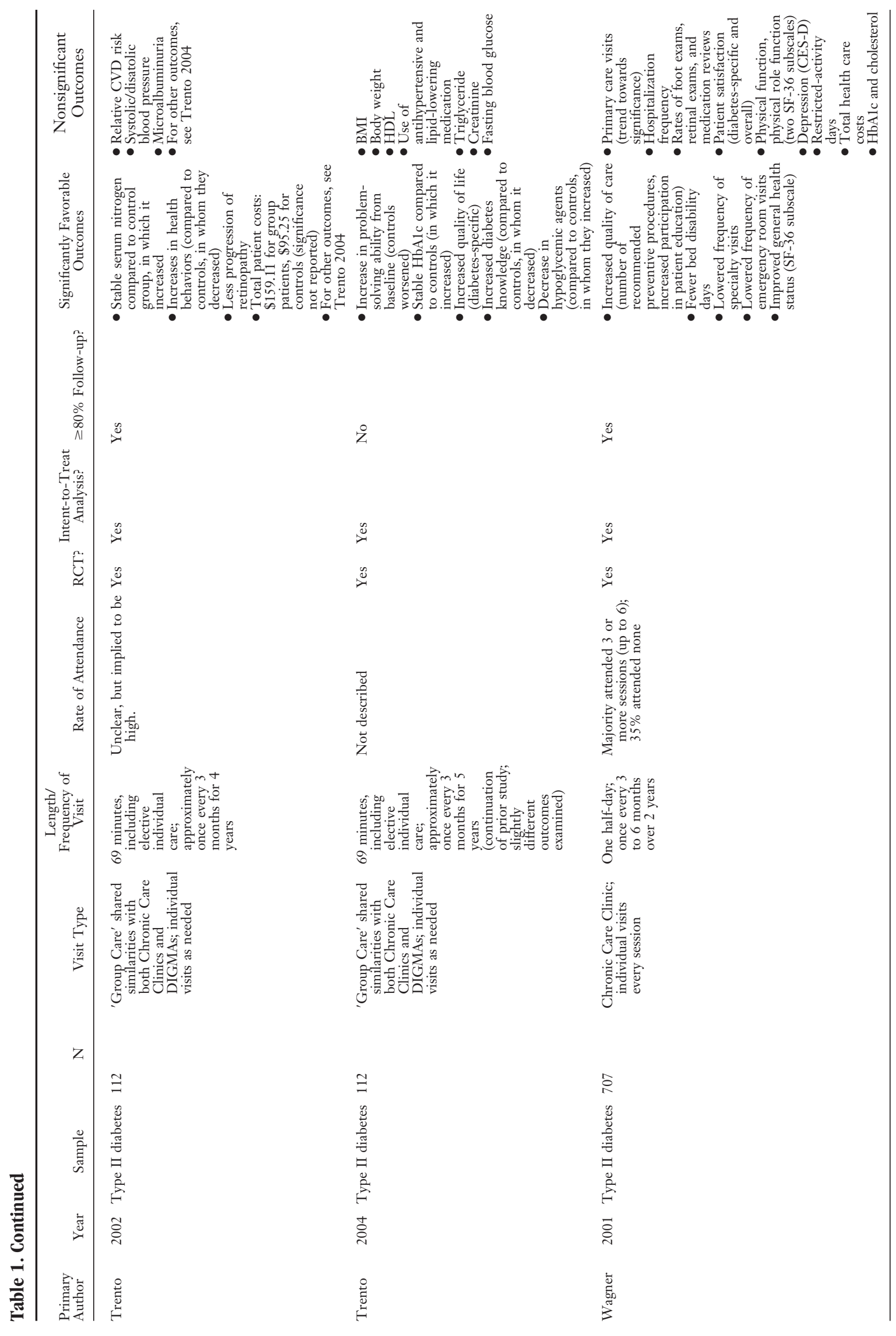




to demonstrate this heterogeneity and so that readers may evaluate the strength of evidence presented. We chose to proceed with a qualitative review, as it was impossible to complete a systematic review based on SORT criteria ${ }^{39}$ because of the multiplicity of outcomes and sub-outcomes (ie, different aspects of patient satisfaction) measured in each study. We encourage the reader to refer to the table frequently, as it will facilitate the navigation of information and allow more meaningful comparisons and conclusions.

\section{Results}

\section{Patient Satisfaction}

Most studies examining patient satisfaction found that this outcome improved in those subjects receiving group visit interventions.

The first controlled evaluation of group visits examined their effectiveness in well-baby consultations. Osborn and Wooley ${ }^{40}$ found that although patient satisfaction did not significantly differ between groups, patients preferred the group visit format because of the benefits provided by group interaction.

Clancy et al studied 120 economically disadvantaged patients with type 2 diabetes. Group visit participants demonstrated increased levels of trust in their physician compared with controls; there were also trends toward significance pertaining to perceptions of better coordination of care, community orientation, and cultural competency. There were no differences, however, in perceptions of first contact care, longitudinal/ongoing care, comprehensive care, or family-centered care. ${ }^{30-32}$ These findings are supported by Beck et al, as well as Scott et $\mathrm{al}$, who found greater levels of satisfaction in chronically ill, high-using older patients participating in group visits. Patients appreciated the physician's unhurriedness, time spent with the physician, and overall quality of care. ${ }^{34,36}$ Sadur et al also found significantly higher levels of patient satisfaction with diabetes-specific care in the intervention group. ${ }^{35}$

In a qualitative study exploring the feasibility of conducting group visits with low-income women at an inner city clinic, Miller et al reported that factors contributing to patient satisfaction included personalized attention, advice from others in the group, self-management education, and increased access to prescription refills and examinations. $\mathrm{Pa}-$ tients who attended more sessions reported higher levels of satisfaction. ${ }^{41}$

Two large randomized studies involving diabet$\mathrm{ics}^{42}$ and frail elderly at high risk for hospitaliza$\operatorname{tion}^{43}$ found no significant differences between controls and group visit participants regarding patient satisfaction. These 2 studies, however, featured half-day group visits with long intervals between sessions ( 3 to 4 and 3 to 6 months), and attendance rate was poor in both; among diabetics, $35 \%$ of intervention subjects attended no sessions and participation was even lower in the elder study. In both studies, increased participation was associated with higher levels of patient satisfaction.

In the only study of patient satisfaction for those attending DIGMAs, Noffsinger and Atkins found that the overall mean patient satisfaction score was 4.7 out of $5 .{ }^{16}$

\section{Health Service Utilization}

The majority of studies measuring health service utilization demonstrated decreased utilization of some specific health services. These were emergency department visits, visits to specialists, and hospitalization rates.

Beck et al found that chronically ill older adults attending CHCCs made fewer "same day" internal medicine visits, emergency department visits, imaging tests, visits to specialists and had less repeat hospital admissions. There were no differences between groups regarding number of laboratory tests, medications prescribed, admissions to skilled nursing facilities, or visiting nurse services. ${ }^{34}$ Similarly, the study by Scott et al of chronically ill elderly revealed that CHCC patients with chronic illness had fewer emergency department visits, hospital admissions, and professional services than controls. Although there were no differences between study groups in utilization of outpatient hospital visits, observation unit admissions, and home health visits, subjects with higher attendance rates had fewer clinic visits, pharmacy fills, and skilled nursing facility admissions. ${ }^{36}$ Miller's small feasibility study on low-income, chronically ill women demonstrated a significant decrease in emergency and urgent care visits during the intervention compared with a similar period before the intervention; there were no differences, however, in regards to overall frequency of clinic visits. ${ }^{41}$

Sadur's investigation of diabetic group visits demonstrated fewer hospitalizations and non-phy- 
sician (eg, nutritionist) visits than controls; however, there were no differences in regards to physician visits (which approached significance), urgent care visits, emergency visits, or visits to the optometrist or ophthalmologist. ${ }^{35}$ Wagner's investigation of diabetic patients demonstrated lower frequency of specialty and emergency department visits, but no significant differences in primary care visits (which approached significance), hospitalization frequency, rates of foot and retinal exams, or medication reviews. ${ }^{42}$

Maizels reported that a group visit intervention designed for chronic headache resulted in dramatically lower outpatient and emergency department utilization, although significance was not assessed. ${ }^{44}$ The study done by Blumenfeld and Tischio of a group visit program for headache also reported decreased primary care visits, emergency visits, and CT scans, but neurology visits and use of injected and oral narcotics increased. ${ }^{45}$

Osborn's evaluation of well-child group visits implied no significant differences between intervention subjects and controls regarding illness-related calls, visits to physicians and nurses, or emergency department visits. ${ }^{40}$ These study results may have been due to the low-risk nature of the population, as well as a small sample. Similarly, Coleman, who studied 169 frail elderly at high risk for hospitalization and functional decline, found no differences in health care utilization, including frequency of hospitalization, hospital days, emergency and ambulatory visits, total costs of care, or use of high-risk medications between control and intervention groups. These results, however, may be secondary to low attendance rates, large time interval between visits, and/or the choice of extremely frail elderly at high risk for hospitalization for the study sample. ${ }^{43}$

\section{Quality of Care}

Quality of care improved in all group visits studies measuring this outcome. Wagner, in a study of 707 diabetic patients, reported that group visit participation was positively associated with subjects receiving preventive procedures, having medications reviewed, and having a microalbuminuria test recorded in the diabetes registry. ${ }^{42}$ Similarly, Clancy found that diabetic patients participating in group visits demonstrated improvement in 10 American Diabetes Association processes of care indicators. $^{30-32}$ A study by Sadur showed that poorly controlled diabetics (average HbA1C greater then $8.5 \%)$, who attended group visits significantly increased intake of insulin, sulfonylureas, and metformin compared with controls, ${ }^{35}$ whereas another study of relatively well-controlled type 2 diabetic patients attending group visits demonstrated lowered dosage of hypoglycemic agents than in standard care. ${ }^{37}$

In a study of chronically ill, high-using older adults attending CHCCs, Beck found that group visit participants received more influenza and pneumonia vaccinations compared with controls. ${ }^{34}$ Scott et al found that group visit participants reported significantly higher frequencies of patient education about medication management from pharmacists and self-management techniques from nurses compared with controls. ${ }^{36}$ Finally, Dodds reported that physicians providing group well-child visits covered significantly more American Academy of Pediatrics-recommended content in their education sessions than did control physicians, particularly about safety, nutrition, behavior and development, and sleep. ${ }^{46}$

\section{Healthy Behaviors, Self-Care, and Self-Efficacy}

Investigations exploring the effects of group visits on healthy behaviors and self-efficacy demonstrated mixed results.

Trento et al studied 112 previously diagnosed, non-insulin-treated type 2 diabetics who were randomized to a control arm receiving usual care, and an experimental arm participating in group visits. Group visit patients demonstrated increases in diabetes knowledge and healthy behaviors, whereas both decreased in controls. ${ }^{37}$ Five-year follow-up of this patient cohort revealed that group patients also demonstrated significantly increased problemsolving abilities, in which they were able to identify underlying health problems and potential solutions, compared with controls (in whom this ability decreased). ${ }^{38}$ Sadur found that compared with controls, diabetic group visit patients increased their frequency of blood glucose monitoring, although the proportion of patients monitoring blood glucose did not increase. In addition, there were no differences between groups in regards to self-assessed ease of maintaining an acceptable blood glucose level, frequency of foot self-examinations, or exercise.

Masley reported that subjects with coronary artery disease participating in group visits signifi- 
cantly increased fruit and vegetable intake, as well as utilization of monosaturated cooking oils compared with controls, although there were no differences in regards to total and saturated fat intake. ${ }^{33}$

Sadur found that group visit patients reported significantly higher levels of self-efficacy in controlling diet to lower blood glucose, recognizing and treating hyperglycemia, and maintaining blood glucose when ill, although there were no differences between groups in self-efficacy for following a low-fat diet, exercising regularly, monitoring blood glucose regularly, communicating with physicians, or expressing feelings about diabetes to family and friends. ${ }^{35}$ Scott et al found that patients participating in CHCCs reported significantly higher self-efficacy in communicating with their physician, but no differences between groups in self-efficacy for disease management, doing chores, participating in social or recreational activities, or controlling/managing depression. ${ }^{36}$

\section{Physical Function, Depression, and Quality of Life}

Whereas it seems that group visits increase quality of life, they do not succeed as well at improving physical function and depression.

Trento et al reported improved diabetes specific quality of life for adults with type 2 diabetes participating in group visits, although this improvement did not occur until the second intervention year $^{38}$; in contrast, controls worsened. In a study conducted by Blumenfeld and Tischio, a small prospective cohort of patients attending group visits for the treatment of headache showed improvement in headache-related quality of life and physical function measured with the SF-36 Health Survey (SF-36) at 8 weeks, which was maintained 6 months postintervention. ${ }^{45}$ The evaluation of Osborn and Wooley of well-child group visits found that mothers attending group visits were significantly less likely to say their child had been ill. $^{40}$

Scott et al conducted a study of 294 chronically ill patients ( $>60$ years old) who were high service utilizers, defined as having had 11 or more outpatient visits in the past 18 months. CHCC patients reported a significantly higher overall quality of life, but there were no significant differences between the intervention group and controls regarding physical function [ie, activities for daily living (ADLs) and instrumental ADLs (IADLs)]. ${ }^{36}$ Wag- ner demonstrated that increased group visit participation was positively associated with a reduction in bed disability days and an increase in the general health status subset of the SF-36 compared with usual care among diabetics, but there were no significant improvements in the physical function and physical role function subsets of the SF-36 or depression as measured by the Centers for Epidemiologic Studies Depression Scale (CES-D). ${ }^{42}$

Beck et al found no significant differences between group patients and controls in functional status (ie, mobility, ADLs, and IADLs) or depression. ${ }^{34}$ Finally, Coleman et al found that frail elderly at high risk of hospitalization participating in group visits demonstrated no significant improvements in depression or physical function (SF-36) compared with the control group. Although group patients had decreased urinary incontinence compared with controls at a 12-month follow-up, this difference had disappeared at 24 months. ${ }^{43}$

\section{Disease-specific Outcomes}

The group visit literature discussed several disease outcomes, including coronary artery disease, headache, obesity, and type 2 diabetes; again, the results have been mixed.

In a 12-month randomized controlled trial of a group visit program for cardiovascular disease, Masley et al found that group visit participants achieved significant reductions in low-density lipoprotein (LDL) levels compared with baseline, although the difference in LDL reduction between groups was not significant. No differences were noted for HbA1C, triglyceride levels, high-density lipoprotein (HDL), or total cholesterol/HDL ratio. ${ }^{33}$ In a non-controlled trial, Power demonstrated a $20 \%$ success rate (defined as a loss of 10 pounds or greater) among obese group visit participants over 5 years, compared with a $5 \%$ success rate to obesity interventions that had been thus far reported in the literature. In addition, he reported a $20 \%$ reduction in blood pressure among obese hypertensive patients and an $80 \%$ reduction in cholesterol among obese hypercholesterolemic patients, although significance was not assessed. ${ }^{47}$

Trento et al found that type 2 diabetics participating in group visits achieved stable HbA1C and serum urea nitrogen, whereas those receiving individualized treatment demonstrated increases in 
both outcomes. Diabetic retinopathy improved among group patients but worsened in controls. ${ }^{37}$ There were no differences in body mass index, body weight, relative cardiovascular disease risk, blood pressure, HDL, triglyceride, or creatinine between the intervention and control group. Power found that among diabetic group visit participants, satisfactory control of blood glucose levels (80-120 $\mathrm{mg} / \mathrm{dL}$ ) was achieved in $\sim 35 \%$ of patients by their fourth group session, whereas blood glucose levels among diabetics receiving individual visits were not significantly altered after 1 year. ${ }^{48}$ No differences in body weight were found between groups. In the diabetes group visits study conducted by Sadur et al, HbA1C levels declined significantly by $1.3 \%$ for those attending group visits, compared with $0.22 \%$ for controls at 6 months. In addition, there was a significant decrease in the average home blood glucose level compared with controls. At 12 months, however, there were no significant differences between groups in regards to HbA1C. ${ }^{35}$

Clancy et al found no differences between group visit patients and controls in lipid profiles and $\mathrm{HbA1C}^{30-32}$; similarly, Wagner reported no significant differences between diabetic patients receiving usual care or group care in HbA1C and cholesterol. However, frequency of group visit attendance was positively correlated with reduced HbA1C levels and improved cholesterol. ${ }^{42}$

Blumenfeld and Tischio found that $92 \%$ of patients attending group visits for headache reported subjective improvement of symptoms at the completion of the program; this was maintained at 6 months. This intervention offered one 2-hour group visit led by a neurologist and followed by a very comprehensive and lengthy individual visit at 2 and 8 weeks led by a nurse practitioner. The individual visit included careful case management of headache patients with medications and lifestyle modifications, and collaboration as needed with a psychiatrist for depression comorbidity, biofeedback for stress management, pain management for patients with pain comorbidities such as fibromyalgia, and anesthesia services for detoxification from narcotics. ${ }^{45}$ Similarly, Maizels et al reported a decrease in the frequency of severe headaches among group visit patients, although significance was not assessed. ${ }^{44}$ These 2 studies were uncontrolled prospective cohorts.

\section{Satisfaction of Physicians Participating in Group Visits}

Physician satisfaction, when measured, has been shown to be high across all group visit studies. Beck et al found that all physicians who participated in CHCCs reported that they greatly enjoyed and were extremely satisfied with their ability to treat group visit patients. ${ }^{34}$ Blumenfeld and Tischio found that of 77 primary care physicians who responded to a satisfaction survey after their patients attended a group visit for headache conducted by a neurologist/nurse practitioner team, $86 \%$ were satisfied overall, approximately $10 \%$ were neutral, and less than $4 \%$ were somewhat dissatisfied. ${ }^{45}$

Group visits have been shown to increase physician productivity, which may in turn increase physician satisfaction. In a study of 4 pilot DIGMA programs that took place weekly for 6 weeks, Noffsinger and Atkins found that physicians were able to see an average of 41.8 patients per week in 5.5 hours of physician time. If these physicians had used those hours to provide traditional care, they would have been only able to see an average of 16.3 patients each. Overall, the average combined increase in productivity was $256.4 \% .{ }^{16}$ This was associated with greater levels of qualitatively reported physician satisfaction, specifically in relation to spending more time with patients without increasing total time worked.

\section{Cost of Care}

To date, most group visits have not demonstrated a decrease in total cost of care. All total cost of care analyses was done in fully capitated health-maintenance organizations (HMOs) or a nationalized health care system.

The study conducted by Beck et al of chronically ill high utilizers was the only quality study that demonstrated a decrease in total cost of care: $\$ 14.79$ per participant per month in CHCC patients, compared with controls. ${ }^{34}$

Maizels et al reported that prescription costs were reduced in previously high utilizers of triptans after a group visit intervention, and the overall cost of the program to the physicians' practice was reduced by $\$ 18,757$. This was based on a theoretical cost of $\$ 60$ for office visits and $\$ 100$ for emergency department visits, ${ }^{44}$ although significance was not assessed.

Scott et al assessed total costs, including pharmacy, hospital, professional, and health plan termi- 
nation costs, for all CHCC patients compared with controls and did not find the cost savings significant. Patients attending CHCCs, however, demonstrated significantly lower emergency department costs compared with controls. ${ }^{36}$

The data of Coleman et al implied no significant reduction in total or pharmacy costs secondary to the group visit intervention. ${ }^{43}$ Similarly, Masley et al found that the intervention group had lower per-member-per-month expenses than a control group that received the National Cholesterol Education Program's Step II-III diet plan; however, the cost difference was not statistically significant. ${ }^{33}$

Trento et al examined 2 types of direct coststhose paid by the Italian National Health Service for staff, educational materials, clinical procedures, and pharmacological treatment, and those incurred by patients and their caregivers, including transportation costs and opportunity-cost value of time. ${ }^{37}$ Only costs for pharmacological treatment decreased among group visit patients compared with controls. In fact, group visits were more costly than control visits, and the researchers concluded that an each per-point increase in quality of life (as calculated from modified Diabetes Quality of Life Questionnaires) would cost an additional \$2.12 per patient over usual care.

\section{Discussion}

The interpretation of these results is difficult, due in part to differences in study applications of the group visit model. As depicted in Table 1, interventions differ widely in structure, processes of care, and research quality. Our summary of results, however, is consistent with the conclusions of the major and higher quality studies. ${ }^{30,33,34,36,37,42,43}$

Studies differed in group visit model, education content, presentation style, population treated, and length and frequency of program sessions (Table 1). In many cases, different instruments were used to measure the same outcome (ie, physical function). Some scales were general, whereas some were disease-specific (ie, quality of life). In addition, individual visits were not always provided, and if provided, differed in length and comprehensiveness. In addition, telephone follow-ups were sometimes added to the intervention. Although flexibility is one of the main advantages of the group visit model (because it can be altered to fit various patient populations, specific physician practices/orga- nizations, and a number of health care delivery systems), the resultant variability limits the ability to compare studies and to generalize the results of a given study to other contexts.

There seems to be, however, a consensus among the data that group visits improve satisfaction, quality of life, and quality of care indicators. We believe this is due to the expanded time component that provides greater interaction between patients and providers and affords physicians time to apply and monitor specific chronic care guidelines. On the other hand, healthy behavior and self-efficacy improvement is much more complex and linked to the facilitator's skills in motivational interviewing, ${ }^{49}$ presence of behavior-specific objectives, and patients confidence level. ${ }^{18}$

In general, however, patients seem to incur fewer visits to emergency rooms and specialists, implying better management and self-management skills. The lack of improvement of function in 3 major studies ${ }^{34,36,43}$ may have been due to the poor match between instrument and population. For example, it may have been more appropriate to use the ADLs and IADLs scales with the very sick frail elderly $^{43}$ and use the SF-36 for chronically ill elderly. ${ }^{34,36}$ In addition, none of the studies involved an experiential component, such as exercise, to improve physical function. It was sobering to see that depression did not improve when measured, because depression is an important and common comorbidity that may have an effect on patient selfmanagement and disease outcomes. ${ }^{50}$ Treatment of depression, however, was not explicitly addressed by any of the studied interventions. Group visits (as any chronic disease management program) need to address and treat depression. Finally, the variability in behavior change and disease outcome may also be linked to the availability and quality of the individual visit. We believe that the individual visit plays an important role in providing patients with personalized feedback by tailoring the education discussion to their specific needs. Therefore, occasional or very short individual visits may be problematic.

If the group visit is to become an adjunctive model for chronic disease management for family physicians, there is an obvious need to delineate and refine the structural elements, process and content of these visits, as well as to delineate the best instruments to use for measuring general and spe- 
cific chronic illness outcomes. In particular, researchers need to note:

\section{Structure}

A. Are individual visits separate from the group or within the group?

B. What is the program length and frequency?

C. What is the nature of the patient cohort (ie, from a physician's practice, from the community, or both)?

D. Is the group composed of the same patients from week to week?

E. What are the population characteristics that determine inclusion in the group?

F. Are there follow-up telephone calls or sessions for "graduates"?

G. Will the group include a mental health practitioner or other providers in addition to a physician/nurse team?

II. Processes of care

A. Is information presented in a didactic lecture or via interactive discussions?

B. Is skill-building experiential (ie, via exercise, breathing and meditation sessions or a potluck meal)?

C. Are motivational interviewing techniques used, specific behavioral goals set, and selfefficacy assessed at each session, as recommended by Lorig and Holman ${ }^{18}$

III. Content

A. What are the details about group education information regarding physiology, disease development, medications, lifestyle interventions (including nutrition, exercise, and stress management), and other aspects of chronic disease self-management?

B. What is the content of the individual visit?

C. Is screening and treatment of depression provided?

D. What is the degree of comprehensiveness of assessment and case management and collaboration with other providers (ie, physical therapists, dietitians, psychotherapists)?

We believe that whereas randomized controlled trials are justifiably the standard in the assessment of causality, the time-intensive nature of group visits requires the choice of highly motivated subjects to enhance participation, at the risk of intro- ducing an element of selection bias. The potential for bias is enhanced due to the impossibility of conducting a blinded study, because patients are, out of necessity, aware of the intervention they will receive. One option is to use willingness to participate in group visits as a criterion for study inclusion and then randomizing all respondents who fit that criterion to the experimental or control arm. It must be noted, however, that many studies demonstrated low attendance rates, particularly if meetings occurred at infrequent intervals or over an extended period (over 1 year) even when patient populations were motivated at the outset. ${ }^{26,34,42,43}$ This phenomenon will affect study conclusions and will require inclusion of a dose-response analysis of data that allows for interpretation of patient results based on the number of group visit sessions attended, in addition to an intent-to-treat analysis.

Most researchers examining the efficacy of the group visit model did so in the context of a fully capitated HMO (ie, Kaiser Permanente, ${ }^{35,36,44,45}$ Group Health Cooperative of Puget Sound ${ }^{3,42,43}$ ) or a nationalized health care system. ${ }^{37,38}$ In these contexts, it is easier to use a multidisciplinary team to conduct group visits and to measure total costs of care and health care utilization, including visits to specialists and emergency rooms and admissions to skilled nursing facilities and hospitals.

Although the cost of care and health care utilization are more important to fully capitated systems, they are also relevant to fee-for-service managed care insurance models. Improvements in disease outcomes and quality of care indicators are attractive to the Medicare and private sector, as well. Financial profitability, however, can be computed by comparing group visits' level of billing and patient numbers to usual care. Several articles have been written that address how to provide group visits in a fee-for-service context. ${ }^{11,51}$ Some of these report a positive profit margin secondary to seeing a larger number of patients in less time $^{11,16}$; this has not been our experience. ${ }^{21}$

\section{Conclusion}

Group visits improve patient and physician satisfaction, quality of care, and quality of life, as well as decrease health care utilization, particularly visits to the emergency department and specialists and sometimes rates of hospitalization or readmission. Their impact on healthy behaviors, self-efficacy, 
and disease outcomes is more variable, probably because of the study differences in structures, processes, and content of care. Their impact on physical function has also been variable, probably due to lack of exercise training and inappropriate choice of measurements. Depression has not improved when measured and has not been specifically addressed as an important comorbidity by this model It is not yet evident that group visits decrease total cost of care.

There is a need to explore the feasibility and reproducibility of this model in primary care practices. Future research may benefit, however, from abandoning old nomenclatures and clearly defining the structure and processes of care, the content of visits, and appropriate outcome measures.

Group visits are a promising approach to chronic care management for the motivated patient. They provide a mechanism for providing time for education combined with medical care in a manner that at least maintains productivity and revenue. The combination of individual medical attention and group education, if well-designed, has the potential to address multiple aspects of patient care in a personalized, tailored fashion but may only be applicable to motivated patients who are willing to invest extra time in their care. Thus, the group visit model is a useful adjuvant to the chronic care model.

\section{References}

1. National Center for Chronic Disease Prevention and Health Promotion. Chronic disease overview. Centers for Disease Control and Prevention, 2003 [Accessed 03/15/04]. Available from:〈http://www. cdc.gov/nccdphp/overview.htm $>$.

2. Mili F, Helmick C, Zack M, Moriarty D. Health related quality of life among adults reporting arthritis: analysis of data from the Behavioral Risk Factor Surveillance System, US, 1996-99. J Rheumatol 2003;30:160-6.

3. Haggerty C, Schulz R, Ness R, Investigators. PEaCHS. Lower quality of life among women with chronic pelvic pain after pelvic inflammatory disease. Obstet Gynecol 2003;102:934-9.

4. Wehler M, Reulbach U, Nichterlein R, et al. Health-related quality of life in chronic pancreatitis: a psychometric assessment. Scand J Gastroenterol 2003;38:1083-9.

5. Cheng L, Cumber S, Dumas C, Winter R, Nguyen $\mathrm{K}$, Nieman L. Health related quality of life in pregeriatric patients with chronic diseases at urban, public supported clinics. Health Qual Life Outcomes 2003;1:63.

6. Wellington M. Stanford Health Partners: rationale and early experiences in establishing physician group visits and chronic disease self-management workshops. J Ambulatory Care Manage 2001;24:10-6.

7. Feldman M. Cluster visits. Am J Nurs 1974;74: 1485-8.

8. Spann SJ. Task Force Report 6. Report on financing the new model of family medicine. Ann Fam Med 2004;2:S1-21.

9. Pennachio DL. Should you offer group visits? Med Econ 2003;80:70-2, 82, 85 .

10. Shute N. That old-time medicine. U.S. News and World Report, 2002;55-8:60-1.

11. Masley S, Sokoloff J, Hawes C. Planning group visits for high-risk patients. Fam Pract Manag 2000; 7:33-7.

12. Walker T. Medical visits get group mentality approach: group appointments can increase efficiency and patient and physician satisfaction. Managed Healthcare 2000;10:10.

13. Thompson E. The power of group visits: improved quality of care, increased productivity entice physicians to see up to 15 patients at a time. Mod Healthcare 2000;30:54.

14. Trilling J. Selections from current literature: focus group technique in chronic illness. Fam Pract 1999; 16:539-41.

15. Jaber R, Braksmajer A, Trilling J. Group visits: models, benefits, and challenges. Fam Pract Manag 2006; $13: 37-40$.

16. Noffsinger EB, Atkins TN. Assessing a group medical appointment program: a case study at Sutter Medical Foundation. Group Pract J 2001;48:42-9.

17. Noffsinger E, Sawyer DR, Scott JC. Group medical visits: a glimpse into the future? (enhancing your practice). Patient Care 2003;37:18-27.

18. Lorig KR, Holman HR. Self-management education: history, definition, outcomes, and mechanisms. Ann Behav Med 2003;26:1-7.

19. Bandura A. Social foundations of thought and action. Englewood Cliffs (NJ): Prentice-Hall, 1986.

20. Marks R, Allegrante JP, Lorig K. A review and synthesis of research evidence for self-efficacy-enhancing interventions for reducing chronic disability: implications for health education practice (Part I). Health Promotion Pract 2005;6:37-43.

21. Jaber R, Braksmajer AS, Trilling J. Group visits: our experience with this adjunctive model to chronic care management. Internet J Fam Pract 2005;4. Available from: 〈http://www.ispub.com〉.

22. Noffsinger EB. Answering physician concerns about drop-in group medical appointments. Group Pract J 1999;48:14-21.

23. Noffsinger EB. Benefits of drop-in group medical 
appointments (DIGMAs) to physicians and patients. Group Pract J 1999;48:21-8.

24. Dreffer D. Group visits hit the road. Fam Pract Manag 2004;11:39-42.

25. Houck S, Kilo C, Scott J. Improving patient care: group visits 101. Fam Pract Manag 2003;10:66-8.

26. Scott J, Gade G, McKenzie M, Venohr I. Cooperative health care clinics: a group approach to individual care. Geriatrics 1998;53:68-74.

27. Escobar GJ, Braveman PA, Ackerson L, et al. A randomized comparison of home visits and hospitalbased group follow-up visits after early postpartum discharge. Pediatrics 2001;108:719-27.

28. Trento M, Passera P, Tomalino M, et al. Group visits improve metabolic control in type 2 diabetes. Diabetes Care 2001;24:995-1000.

29. Coleman E, Eilertsen T, Kramer A, Magid D, Beck A, Conner D. Reducing emergency visits in older adults with chronic illness: a randomized, controlled trial of group visits. Eff Clin Pract 2001;4:49-57.

30. Clancy DE, Brown SB, Magruder KM, Huang P. Group visits in medically and economically disadvantaged patients with type 2 diabetes and their relationships to clinical outcomes. Top Health Inf Manage 2003;24:8-15.

31. Clancy DE, Cope DW, Magruder KM, Huang P, Salter KH, Fields AW. Evaluating group visits in an uninsured or inadequately insured patient population with uncontrolled type 2 diabetes. Diabetes Educ 2003;29:292-301.

32. Clancy DE, Cope DW, Magruder KM, Huang P, Wolfman TE. Evaluating concordance to American Diabetes Association standards of care for type 2 diabetes through group visits in an uninsured or inadequately insured patient population. Diabetes Care 2003;26:2032-6.

33. Masley S, Phillips S, Copeland JR. Group office visits change dietary habits of patients with coronary artery disease. J Fam Pract 2001;50:235-9.

34. Beck A, Scott J, Williams $P$, et al. A randomized trial of group outpatient visits for chronically ill older HMO members: the cooperative health care clinic. J Am Geriatr Soc 1997;45:543-9.

35. Sadur CN, Moline N, Costa M, et al. Diabetes management in a health maintenance organization: efficacy of care management using cluster visits. Diabetes Care 1999;22:2011.

36. Scott JC, Conner DA, Venohr I, et al. Effectiveness of a group outpatient visit model for chronically ill older health maintenance organization members: a 2-year randomized trial of the cooperative health care clinic. J Am Geriatr Soc 2004;52:1463-70.

37. Trento M, Passera P, Bajardi M, et al. Lifestyle intervention by group care prevents deterioration of type II diabetes: a 4-year randomized controlled clinical trial. Diabetologia 2002;45:1231-9.

38. Trento M, Passera P, Borgo E, et al. A 5-year randomized controlled study of learning, problem solving ability, and quality of life modifications in people with type 2 diabetes managed by group care. Diabetes Care 2004;27:670-5.

39. Ebell MH, Siwek J, Weiss BD, et al. Strength of Recommendation Taxonomy (SORT): A patientcentered approach to grading evidence in the medical literature. J Am Board Fam Pract 2004;17:59-67.

40. Osborn LM, Wooley FR. Use of groups in well child care. Pediatrics 1981;67:701-6.

41. Miller D, Zantop V, Hammer H, Faust S, Grumbach K. Group medical visits for low-income women with chronic disease: a feasibility study. J Womens Health 2004;13:217-25.

42. Wagner E, Grothaus L, Sandhu N. Chronic care clinics for diabetes in primary care. Diabetes Care 2001;25:695-700.

43. Coleman E, Grothaus L, Sandhu N, Wagner E. Chronic care clinics: a randomized controlled trial of a new model of primary care for frail older adults. J Am Geriatr Soc 1999;47:775-83.

44. Maizels M, Saenz V, Wirjo J. Impact of a groupbased model of disease management for headache. Headache 2003;43:621-7.

45. Blumenfeld A, Tischio M. Center of excellence for headache care: group model at Kaiser Permanente. Headache 2003;43:431-40.

46. Dodds M, Nicholson L, Muse B, Osborn LM. Group health supervision visits more effective than individual visits in delivering health care information. Pediatrics 1992;91:668-70.

47. Power L. Group office visits in obesity. J Fla Med Assoc 1992;79:392-5.

48. Power L. Group approach to diabetes care: a preliminary note. Postgrad Med 1983;73:211-6.

49. Rubak S, Sandbaek A, Lauritzen T, Christensen B. Motivational interviewing: a systematic review and meta-analysis. Br J Gen Pract 2005;55:305-12.

50. Kupfer DJ, Frank E. Comorbidity in depression. Acta Psychiatr Scand Suppl 2003;s418:57-60.

51. Masley S. Group visit templates for osteoporosis, asthma, and hypertension. Preconference on Group Medical Visits. Rye (NY): Northeast Society of Teachers of Family Medicine, 2004. 